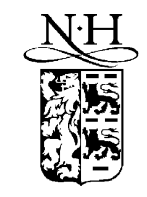

Available online at www.sciencedirect.com

ELSEVIER

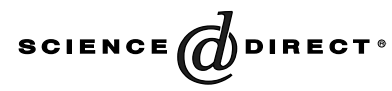

Applied Numerical Mathematics 48 (2004) 307-322

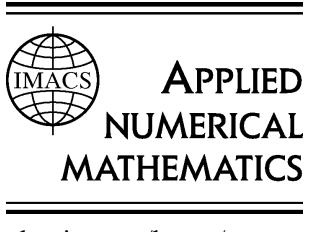

www.elsevier.com/locate/apnum

\title{
Geometric space-time integration of ferromagnetic materials
}

\author{
Jason Frank ${ }^{1}$
}

CWI, P.O. Box 94079, 1090 GB Amsterdam, The Netherlands

\begin{abstract}
The Landau-Lifshitz equation (LLE) governing the flow of magnetic spin in a ferromagnetic material is a PDE with a noncanonical Hamiltonian structure. In this paper we derive a number of new formulations of the LLE as a partial differential equation on a multisymplectic structure. Using this form we show that the standard central spatial discretization of the LLE gives a semi-discrete multisymplectic PDE, and suggest an efficient symplectic splitting method for time integration. Furthermore we introduce a new space-time box scheme discretization which satisfies a discrete local conservation law for energy flow, implicit in the LLE, and made transparent by the multisymplectic framework.
\end{abstract}

๑ 2003 IMACS. Published by Elsevier B.V. All rights reserved.

Keywords: Ferromagnetic materials; Landau-Lifshitz equation; Multisymplectic structure; Geometric integration

\section{Hamiltonian structure of the Landau-Lifshitz equation}

This paper addresses the Landau-Lifshitz equation (LLE) as a nonlinear wave equation supporting solitons and stable magnetic vortices, as considered, e.g., in [5,19,23]. The LLE governs the flow of magnetic spin in a ferromagnetic material. At a point $\mathbf{x} \in \mathrm{R}^{d}$ the $\operatorname{spin} \mathbf{m}(\mathbf{x}, t)=\left(m_{1}, m_{2}, m_{3}\right)^{\mathrm{T}}$ in Cartesian coordinates satisfies

$$
\mathbf{m}_{t}=\mathbf{m} \times[\Delta \mathbf{m}+D \mathbf{m}+\Omega],
$$

where $\Delta$ is the Laplacian operator in $\mathrm{R}^{d}, D=\operatorname{diag}\left(d_{1}, d_{2}, d_{3}\right)$ models anisotropy in the material, and $\Omega$ is an external magnetic field.

In applications in micromagnetics, the LLE may additionally include a nonlocal term, a spin magnitude-preserving Gilbert damping term, as well as a coupling terms to a dynamic external field governed by Maxwell's equations, see [6].

E-mail address: jason@ cwi.nl (J. Frank).

${ }^{1}$ Funding from an NWO Innovative Research Grant is gratefully acknowledged. 
The LLE can be written in the form of a Hamiltonian PDE with a nonlinear Lie-Poisson structure (see, e.g., [22,8]). The general form of a Hamiltonian PDE is

$$
\mathbf{y}_{t}=B(\mathbf{y}) \frac{\delta H}{\delta \mathbf{y}},
$$

where $\mathbf{y}(\mathbf{x}, t) \in \mathrm{R}^{p}, H$ is a functional, $\frac{\delta H}{\delta \mathbf{y}}$ is the vector of variational derivatives of $H$ with respect to $\mathbf{y}$, and $B(\mathbf{y})$ is a Poisson structure matrix, i.e., a skew-symmetric matrix operator satisfying the Jacobi identity (see [22]). If $B(\mathbf{y})$ is a Poisson structure matrix, continuous with respect to $\mathbf{y}$, there is a local change of variables $\overline{\mathbf{y}}=\overline{\mathbf{y}}(\mathbf{y})$ such that the structure assumes a canonical form

$$
\frac{\delta \overline{\mathbf{y}}}{\delta \mathbf{y}} B(\mathbf{y}){\frac{\delta \overline{\mathbf{y}}^{\mathrm{T}}}{\delta \mathbf{y}}}^{\mathrm{y}}=J=\left[\begin{array}{ccc}
0 & 0 & 0 \\
0 & 0 & I_{p_{1}} \\
0 & -I_{p_{1}} & 0
\end{array}\right],
$$

where $p=2 p_{1}+p_{2}$ and $I_{p_{1}}$ is the $p_{1}$-dimensional identity matrix. Expressed in the new variables, the Hamiltonian system (2) becomes

$$
\overline{\mathbf{y}}_{t}=J \frac{\delta H(\overline{\mathbf{y}})}{\delta \overline{\mathbf{y}}} .
$$

It is obvious from the structure of $J$ that the dependent variables $\bar{y}_{1}, \ldots, \bar{y}_{p_{2}}$ are constants of motion for any Hamiltonian $H$.

For (1) the Hamiltonian functional is the total energy

$$
H=\frac{1}{2} \int\left|\nabla_{\mathbf{x}} \mathbf{m}\right|^{2}+\mathbf{m} \cdot D \mathbf{m}+2 \Omega \cdot \mathbf{m} \mathrm{d} \mathbf{x},
$$

and the Poisson structure is

$$
B(\mathbf{m})=\hat{\mathbf{m}}=\left[\begin{array}{ccc}
0 & -m_{3} & m_{2} \\
m_{3} & 0 & -m_{1} \\
-m_{2} & m_{1} & 0
\end{array}\right],
$$

which is related to the Poisson structure of the free rigid body [16].

If the spin is alternatively represented in the coordinates $\overline{\mathbf{m}}=\left(m_{\ell}, m_{\theta}, m_{z}\right)^{\mathrm{T}}$,

$$
m_{\ell}=\sqrt{m_{1}^{2}+m_{2}^{2}+m_{3}^{2}}, \quad m_{\theta}=\tan ^{-1} \frac{m_{2}}{m_{1}}, \quad m_{z}=m_{3},
$$

where $\tan ^{-1}$ denotes the angle $\left(m_{1}, m_{2}\right)$ makes with the $m_{1}$ axis, then the Poisson structure takes the canonical form (3) with $p_{1} \equiv p_{2} \equiv 1$, which shows that the spin length $m_{\ell}=|\mathbf{m}|$ is a conserved quantity. Indeed, we have

$$
\frac{\partial}{\partial t}|\mathbf{m}|^{2}=2 \mathbf{m} \cdot \mathbf{m}_{t}=2 \mathbf{m} \cdot\left(\mathbf{m} \times \frac{\delta H}{\delta \mathbf{m}}\right)=0,
$$

for any $H$; that is, $|\mathbf{m}|^{2}$ is a Casimir of (5).

The polar coordinates (6) are well defined except for $m_{1}=m_{2}=0$, for which the spin is aligned with the $m_{3}$ axis. The degenerate case can be treated by defining a local chart with, for example, $m_{\ell}, m_{y}=m_{2}$ and $m_{\phi}=\tan ^{-1}\left(m_{1} / m_{3}\right)$. In this paper we will always assume that locally either $m_{1}$ or $m_{2}$ is nonzero. Although this assumption is crucial for the analysis, the numerical methods developed here are globally defined, making no use of local charts. 
Assuming $D$ and $\Omega$ are independent of $t$ and $x,(1)$ is time- and space-translation invariant, implying the conservation of the total energy (4) and total momentum (given here for $m_{\ell} \equiv 1$ ):

$$
P=\int \frac{1}{1+m_{3}}\left(m_{1} \nabla_{\mathbf{x}} m_{2}-m_{2} \nabla_{\mathbf{x}} m_{1}\right) \mathrm{d} \mathbf{x} .
$$

Both global invariants are consequences of related local conservation laws. For example, in the simplified case: $\{D=I, \Omega=0, d=1\}$, the energy and momentum conservation laws become,

$$
\begin{aligned}
& e_{t}+f_{x}=0, \quad e=\frac{1}{2} \mathbf{m} \cdot \mathbf{m}_{x x}, \quad f=\frac{1}{2}\left(\mathbf{m}_{x} \cdot \mathbf{m}_{t}-\mathbf{m} \cdot \mathbf{m}_{x t}\right), \\
& a_{t}+b_{x}=0, \quad a=\frac{1}{2}\left(m_{3} m_{\theta x}-m_{\theta} m_{3 x}\right), \quad b=\frac{1}{2}\left(m_{\theta} m_{3 t}-m_{3} m_{\theta t}-\left|\mathbf{m}_{x}\right|^{2}\right) .
\end{aligned}
$$

These conservation laws can be integrated over the domain of interest and under appropriate (for example, periodic) boundary conditions, imply the invariance of the total integral. For $\Omega=0$, (1) is also timereversible.

In numerical simulations of the Landau-Lifshitz and related equations, it is crucial to preserve the relation (7). A number of strategies for doing so are encountered in the literature. A general numerical integrator cannot be expected to do this automatically, making it necessary to either impose the condition as a constraint, or to repeatedly project the solution onto the constraint manifold [4]. However, a number of results under the heading of "geometric integration" techniques (see [9]) can be used to construct integrators that automatically preserve the spin magnitude. First, it is well known that the class of GaussLegendre Runge-Kutta methods preserves any quadratic invariant such as the spin magnitude (and the total energy!). The implicit midpoint method is quite common in this context; see the work of Monk and Vacus who use a finite element discretization of micromagnetics [20,21]. Second, given that $\mathbf{m}(x, t)$ evolves on the surface of a sphere, one can derive an equivalent formulation of (1) in the Lie-group $\mathrm{SO}(3)$ (see [24] for a Lie-group formulation of the LLE) and apply Lie-group integrators, as in [10,13]. Third, since the spin magnitude is a Casimir of the Poisson matrix (5), any Poisson integrator will conserve it by definition. In [7] time-reversible, energy conserving, and Poisson integrators were compared against standard methods for the lattice Landau-Lifshitz equation.

The use of geometric integrators places an additional constraint on the discrete phase space of the numerical solution, eliminating some of the freedom ordinary methods have to wander away from geometric structures such as invariant manifolds. On the other hand, the Hamiltonian structure discussed above is really associated with purely temporal quantities. For PDEs, this implies that some integrals over space are well-conserved whereas the local character of the PDE is not addressed. For instance, although the total energy and momentum may be nearly conserved under a symplectic integrator, the flow of energy and momentum from one point in space to another due to the implied conservation laws (9) and (10) is masked by integration. Recent activity has focused on spatio-temporal Hamiltonian structure and multisymplectic PDEs, which do address such local conservation properties. In this paper we propose a new space-time discretization of the LLE which exactly conserves a discrete analog of the implicit energy conservation law (9). We will focus on the case of one spatial dimension $d \equiv 1$, although most of what is said carries over to higher dimensions as well. 


\section{Review of linear multisymplectic structure}

In this section we review some of the implications of multisymplectic structure in the case of linear symplectic forms. In the subsequent section we will generalize these ideas to the nonlinear Poisson case of the Landau-Lifshitz equation. For a full discussion of multisymplectic geometry, see the papers of Bridges [1,2] and Marsden et al. [14].

Given a variational description of a continuous dynamical system (see, e.g., Lanczos [11])

$$
0=\delta \iint \mathcal{L}\left(u, u_{t}, u_{x}\right) \mathrm{d} t \mathrm{~d} x,
$$

the equation of motion is formally given by

$$
-\partial_{t} \frac{\partial \mathcal{L}}{\partial u_{t}}-\partial_{x} \frac{\partial \mathcal{L}}{\partial u_{x}}+\frac{\partial \mathcal{L}}{\partial u}=0
$$

The corresponding Hamiltonian description introduces a conjugate variable $v$ related to the temporal derivative $u_{t}$ by

$$
v \equiv \frac{\partial \mathcal{L}}{\partial u_{t}}
$$

which we assume to define an invertible relationship $u_{t}=u_{t}(v)$. Then the Hamiltonian is defined via a Legendre transformation

$$
H(u, v)=\int v u_{t}(v)-\mathcal{L}\left(u, u_{t}(v), u_{x}\right) \mathrm{d} x
$$

The variational derivatives of $H$ are prescribed to satisfy the original equation of motion (11) and the definition of the conjugate variable $v$ :

$$
\begin{aligned}
& \frac{\delta H}{\delta u}=\partial_{x} \frac{\partial \mathcal{L}}{\partial u_{x}}-\frac{\partial \mathcal{L}}{\partial u}=-\partial_{t} v, \\
& \frac{\delta H}{\delta v}=u_{t}(v)+v u_{t}^{\prime}(v)-\frac{\partial \mathcal{L}}{\partial u_{t}} u_{t}^{\prime}(v)=\partial_{t} u,
\end{aligned}
$$

or, with $\mathbf{y}=(u, v)^{\mathrm{T}}$,

$$
J \mathbf{y}_{t}=\frac{\delta H}{\delta \mathbf{y}}, \quad J=\left[\begin{array}{cc}
0 & -1 \\
1 & 0
\end{array}\right] .
$$

A space-time analog of this procedure yields a multisymplectic structure as follows [1]. A second conjugate variable $w$ is introduced, this time with respect to the spatial derivative $u_{x}$ :

$$
w \equiv \frac{\partial \mathcal{L}}{\partial u_{x}} .
$$

Again we assume this to define an invertible relation $u_{x}=u_{x}(w)$, and a new Hamiltonian is defined by a Legendre transformation with respect to both $v$ and $w$ :

$$
S(u, v, w)=v u_{t}+w u_{x}-\mathcal{L}\left(u, u_{t}(v), u_{x}(w)\right) .
$$

The partial derivatives of $S$ with respect to $(u, v, w)$ are prescribed to satisfy the equation of motion (11) as well as the definitions of $v(12)$ and $w(14)$ : 


$$
\begin{aligned}
& \frac{\partial S}{\partial u}=-\frac{\partial \mathcal{L}}{\partial u}=-\partial_{t} v-\partial_{x} w, \\
& \frac{\partial S}{\partial v}=u_{t}(v)+v u_{t}^{\prime}(v)-\frac{\partial \mathcal{L}}{\partial u_{t}} u_{t}^{\prime}(v)=\partial_{t} u, \\
& \frac{\partial S}{\partial w}=u_{t}(w)+w u_{x}^{\prime}(v)-\frac{\partial \mathcal{L}}{\partial u_{x}} u_{x}^{\prime}(v)=\partial_{x} u,
\end{aligned}
$$

resulting in the form, with $\mathbf{z}=(u, v, w)^{\mathrm{T}}$,

$$
K \mathbf{z}_{t}+L \mathbf{z}_{x}=\frac{\partial S}{\partial \mathbf{z}}
$$

where

$$
K=\left[\begin{array}{ccc}
0 & -1 & 0 \\
1 & 0 & 0 \\
0 & 0 & 0
\end{array}\right], \quad L=\left[\begin{array}{ccc}
0 & 0 & -1 \\
0 & 0 & 0 \\
1 & 0 & 0
\end{array}\right]
$$

Eq. (15) with $K$ and $L$ skew-symmetric matrices defines a PDE on a multisymplectic structure. The theory of such systems has been developed by Bridges [1] and Marsden et al. [14].

Some immediate consequences of multisymplectic structure are summarized below:

Conservation law of symplecticity. If $d \mathbf{y}$ is a solution of the variational equation associated with (13), then the symplectic two-form is globally conserved: $\partial_{t} \frac{1}{2} \int \mathrm{d} \mathbf{y} \wedge J \mathrm{~d} \mathbf{y} \mathrm{d} x=0$. Analogously, if $\mathrm{d} \mathbf{z}$ is a solution of the variational equation associated with (15), a conservation law of symplecticity holds [2]

$$
\partial_{t} \frac{1}{2} \mathrm{~d} \mathbf{z} \wedge K \mathrm{~d} \mathbf{z}+\partial_{x} \frac{1}{2} \mathrm{~d} \mathbf{z} \wedge L \mathrm{~d} \mathbf{z}=0
$$

Integration of this relation over $x$ with appropriate boundary conditions implies the global conservation of symplecticity.

Conservation laws of energy and momentum. Taking the inner product of (13) with $\mathbf{y}_{t}$ yields conservation of total energy $H_{t}=0$ upon integration over space, whereas taking the inner product of (15) with $\mathbf{z}_{t}$ and $\mathbf{z}_{x}$ give local conservation laws of energy and momentum, respectively [1].

$$
\begin{array}{ll}
e_{t}+f_{x}=0, & e=\frac{1}{2} \mathbf{z} \cdot L \mathbf{z}_{x}-S, \quad f=\frac{1}{2} \mathbf{z}_{t} \cdot L \mathbf{z}, \\
a_{t}+b_{x}=0, & a=\frac{1}{2} \mathbf{z}_{x} \cdot K \mathbf{z}, \quad b=\frac{1}{2} \mathbf{z} \cdot K \mathbf{z}_{t}-S .
\end{array}
$$

The multisymplectic structure can be generalized to allow $\mathbf{z}$ dependence in $K$ and $L$, as long as the two-forms associated with $K(\mathbf{z})$ and $L(\mathbf{z})$ are closed, i.e., can be expressed locally as the differentials of one-forms [1,2].

Experience has demonstrated that numerical methods for Hamiltonian systems (13) which take into account the global conservation of total symplecticity and energy exhibit performance superior to standard methods. It is then reasonable to expect that methods which take into account the local conservation laws associated with (15) will also perform well. To this end Marsden et al. [14,12] and Reich and Bridges [25,3] have developed multisymplectic numerical methods.

In this paper we determine a multisymplectic structure for the Landau-Lifshitz equation and discuss related numerical discretizations. 


\section{Multisymplectic structure of the Landau-Lifshitz equation}

To follow the derivation in the previous section, we begin with a variational formulation of the LandauLifshitz equation. We start with a formulation in the coordinates (6) since this gives multisymplectic structure matrices $K$ and $L$ that are constant, simplifying analysis. However for numerical computations the Cartesian components $\left(m_{1}, m_{2}, m_{3}\right)$ are to be preferred, so a constrained multisymplectic structure follows. See [15] for a general framework for constrained multisymplectic theory.

With the spin expressed in the coordinates (6), the canonical equations of motion are

$$
m_{\ell t}=0, \quad m_{\theta t}=\frac{\delta H}{\delta m_{z}}, \quad m_{z t}=-\frac{\delta H}{\delta m_{\theta}},
$$

where the energy (4) takes the form

$$
\begin{gathered}
H=\frac{1}{2} \int m_{\theta x}{ }^{2}\left(m_{\ell}^{2}-m_{z}^{2}\right)+\frac{\left(m_{\ell} m_{\ell x}-m_{z} m_{z x}\right)^{2}}{m_{\ell}^{2}-m_{z}^{2}}+m_{z x}^{2}+d_{1} m_{\ell}^{2} \cos ^{2} m_{\theta}+d_{2} m_{\ell}^{2} \sin ^{2} m_{\theta}+d_{3} m_{z}^{2} \\
+2 \Omega_{1} m_{\ell} \cos m_{\theta}+2 \Omega_{2} m_{\ell} \sin m_{\theta}+2 \Omega_{3} m_{z} \mathrm{~d} x .
\end{gathered}
$$

Since $m_{\ell}(x, t)=m_{\ell}(x, 0)$ is constant in time, it will play the role of a parameter in the variational description. Let $h\left(m_{\theta}, m_{z}, m_{\theta x}, m_{z x}\right)$ be the energy density, that is $H=\int h\left(m_{\theta}, m_{z}, m_{\theta x}, m_{z x}\right) \mathrm{d} x$. Define the action density $\mathcal{L}$ by

$$
\mathcal{L}\left(m_{\theta}, m_{\theta t}\right)=m_{z} m_{\theta t}-h\left(m_{\theta}, m_{z}, m_{\theta x}, m_{z x}\right) .
$$

Introducing new conjugate variables

$$
q_{\theta}=\partial \mathcal{L} / \partial m_{\theta x}=-m_{\theta x}\left(m_{\ell}^{2}-m_{z}^{2}\right), \quad q_{z}=\partial \mathcal{L} / \partial m_{z x}=\frac{m_{\ell} m_{z} m_{\ell x}-m_{\ell}^{2} m_{z x}}{m_{\ell}^{2}-m_{z}^{2}},
$$

the multisymplectic Hamiltonian $S$ is obtained via the Legendre transformation

$$
\begin{aligned}
& S= m_{z} m_{\theta t}+q_{\theta} m_{\theta x}+q_{z} m_{z x}-\mathcal{L} \\
&= q_{\theta} m_{\theta x}+q_{z} m_{z x}+h\left(m_{\theta}, m_{z}, m_{\theta x}, m_{z x}\right) \\
&=\frac{1}{2}\left[-\frac{q_{\theta}^{2}}{m_{\ell}^{2}-m_{z}^{2}}-\frac{q_{z}^{2}}{m_{\ell}^{2}}\left(m_{\ell}^{2}-m_{z}^{2}\right)+\frac{2 m_{\ell} m_{\ell x} m_{z} q_{z}}{m_{\ell}^{2}}+m_{\ell x}{ }^{2}+d_{1} m_{\ell}^{2} \cos ^{2} m_{\theta}+d_{2} m_{\ell}^{2} \sin ^{2} m_{\theta}\right. \\
&\left.\quad+d_{3} m_{z}^{2}+2 \Omega_{1} m_{\ell} \cos m_{\theta}+2 \Omega_{2} m_{\ell} \sin m_{\theta}+2 \Omega_{3} m_{z}\right],
\end{aligned}
$$

and has partial derivatives

$$
\begin{aligned}
\frac{\delta S}{\delta m_{\theta}} & =m_{\ell}^{2}\left(d_{2}-d_{1}\right) \sin m_{\theta} \cos m_{\theta}+m_{\ell}\left(\Omega_{2} \cos m_{\theta}-\Omega_{1} \sin m_{\theta}\right), \\
\frac{\delta S}{\delta m_{z}} & =\frac{q_{z}^{2} m_{z}+q_{z} m_{\ell} m_{\ell x}}{m_{\ell}^{2}}-\frac{m_{z} q_{\theta}^{2}}{\left(m_{\ell}^{2}-m_{z}^{2}\right)^{2}}+d_{3} m_{z}+\Omega_{3}, \\
\frac{\delta S}{\delta q_{\theta}} & =-\frac{q_{\theta}}{m_{\ell}^{2}-m_{z}^{2}}, \\
\frac{\delta S}{\delta q_{z}} & =\frac{-q_{z}\left(m_{\ell}^{2}-m_{z}^{2}\right)+m_{z} m_{\ell} m_{\ell x}}{m_{\ell}^{2}} .
\end{aligned}
$$


The multisymplectic structure has form (15) in coordinates $\mathbf{z}=\left(m_{\theta}, m_{z}, q_{\theta}, q_{z}\right)^{\mathrm{T}}$ with

$$
K=\left(\begin{array}{cccc}
0 & -1 & 0 & 0 \\
1 & 0 & 0 & 0 \\
0 & 0 & 0 & 0 \\
0 & 0 & 0 & 0
\end{array}\right), \quad L=\left(\begin{array}{cccc}
0 & 0 & -1 & 0 \\
0 & 0 & 0 & -1 \\
1 & 0 & 0 & 0 \\
0 & 1 & 0 & 0
\end{array}\right)
$$

The two-forms associated with $K$ and $L$ satisfy the conservation law (16).

The energy and momentum conservation laws for the Landau-Lifshitz equation in these coordinates are given by (9) and (10) with

$$
\begin{aligned}
e & =S+\frac{1}{2}\left(q_{\theta x} m_{\theta}-m_{\theta x} q_{\theta}+q_{z x} m_{z}-m_{z x} q_{z}\right), \\
f & =-\frac{1}{2}\left(q_{\theta t} m_{\theta}-m_{\theta t} q_{\theta}+q_{z t} m_{z}-m_{z t} q_{z}\right), \\
a & =-\frac{1}{2}\left(m_{z x} m_{\theta}-m_{\theta x} m_{z}\right), \\
b & =S+\frac{1}{2}\left(m_{z t} m_{\theta}-m_{\theta t} m_{z}\right) .
\end{aligned}
$$

For numerical computations, the coordinates (6) are impractical because $m_{\theta}$ is undefined for $m_{z}=$ $\pm m_{\ell}$. Alternatively, we can derive a multisymplectic form for the LLE in Cartesian coordinates with a constraint. We rewrite the action density $\mathcal{L}$ in terms of Cartesian coordinates using (6). To preserve the spin length, we add it as a constraint with Lagrange multiplier $\Lambda$

$$
\mathcal{L}=m_{3} \frac{m_{2 t} m_{1}-m_{1 t} m_{2}}{m_{1}^{2}+m_{2}^{2}}-\frac{1}{2}\left(\left|\mathbf{m}_{x}\right|^{2}+\mathbf{m} \cdot D \mathbf{m}+2 \Omega \cdot \mathbf{m}\right)+\Lambda\left(|\mathbf{m}|^{2}-m_{\ell}^{2}\right) .
$$

Define $q_{j}=\partial \mathcal{L} / \partial m_{j x}=-m_{j x}, j=1,2,3$ and the multisymplectic Hamiltonian becomes

$$
S(\mathbf{m}, \mathbf{q})=\frac{1}{2}\left(|\mathbf{q}|^{2}+\mathbf{m} \cdot D \mathbf{m}+2 \Omega \cdot \mathbf{m}\right)-\Lambda\left(|\mathbf{m}|^{2}-m_{\ell}^{2}\right) .
$$

The configuration variable $\mathbf{z}=\left(m_{1}, m_{2}, m_{3}, q_{1}, q_{2}, q_{3}, \Lambda\right)^{\mathrm{T}}$, and the structure matrices $K(\mathbf{z})$ and $L$ are

$$
K(\mathbf{z})=\left[\begin{array}{ccc}
K_{1}(\mathbf{m}) & 0 & 0 \\
0 & 0 & 0 \\
0 & 0 & 0
\end{array}\right], \quad L=\left[\begin{array}{ccc}
0 & I_{3} & 0 \\
-I_{3} & 0 & 0 \\
0 & 0 & 0
\end{array}\right]
$$

where

$$
K_{1}(\mathbf{m})=\left(m_{1}^{2}+m_{2}^{2}\right)^{-1}\left[\begin{array}{ccc}
0 & 0 & -m_{2} \\
0 & 0 & m_{1} \\
m_{2} & -m_{1} & 0
\end{array}\right] .
$$

To check the closedness of the symplectic operator $K(\mathbf{z})$, consider the two-form

$$
\kappa(\mathbf{U}, \mathbf{V})=V_{3} \tan ^{-1} \frac{U_{2}}{U_{1}}
$$

Locally determine orthonormal coordinates such that $z_{1}$ and $z_{2}$ are not both zero, define a one-form $\alpha(\mathbf{z}) \mathbf{V}=\kappa(\mathbf{z}, \mathbf{V})$, i.e., $\alpha(\mathbf{z})=\left(0,0, \tan ^{-1}\left[z_{2} / z_{1}\right]\right)$, and check that $K(\mathbf{z})_{i j}=\frac{\partial \alpha_{j}}{\partial z_{i}}-\frac{\partial \alpha_{i}}{\partial z_{j}}$. 
The equations of motion are

$$
\begin{aligned}
& K_{1}(\mathbf{m}) \mathbf{m}_{t}+\mathbf{q}_{x}=D \mathbf{m}+\Omega-2 \Lambda \mathbf{m}, \\
& -\mathbf{m}_{x}=\mathbf{q}, \\
& 0=|\mathbf{m}(x, t)|^{2}-m_{\ell}(x, 0)^{2} .
\end{aligned}
$$

Premultiplying (26) with $\hat{\mathbf{m}}$ (cf. (5)) gives, for the first term,

$$
\mathbf{m} \times K_{1}(\mathbf{m}) \mathbf{m}_{t}=\left(\begin{array}{c}
\frac{-m_{1} m_{3} m_{3 t}-m_{1} m_{2} m_{2 t}+m_{2}^{2} m_{1 t}}{m_{1}^{2}+m_{2}^{2}} \\
\frac{-m_{2} m_{3} m_{3 t}-m_{2} m_{1} m_{1 t}+m_{1}^{2} m_{2 t}}{m_{1}^{2}+m_{2}^{2}} \\
\frac{m_{1}^{2} m_{3 t}+m_{2}^{2} m_{3 t}}{m_{1}^{2}+m_{2}^{2}}
\end{array}\right)=\mathbf{m}_{t},
$$

where the second equality follows upon substitution of the time derivative of the constraint (28), i.e., $m_{1} m_{1 t}+m_{2} m_{2 t}+m_{3} m_{3 t}=0$. Furthermore, $\mathbf{m} \times 2 \Lambda \mathbf{m}=0$, and substitution of (27) for $\mathbf{q}$ in (26) gives (1).

In the next section we turn to the numerical approximation of (26)-(28). We would just mention again that although the above formulation requires the use of local coordinate charts to handle the case $m_{1}=m_{2}=0$, the methods to be developed in the next two sections are globally defined.

\section{Standard semi-discretization}

Two different approaches to a discrete numerical analog of multisymplectic structure are: that due to Marsden et al. [14,12], which rests on the discretization of the variational formulation, and that due to Reich and Bridges [25,3], which focuses on the Hamiltonian side. In this paper we will consider the latter approach.

In this section we show that the standard spatial discretization of the LLE gives a semi-discrete multisymplectic PDE. Let us introduce a uniform grid with grid-spacing $\xi, x_{i}=i \xi$, and approximations $\mathbf{m}^{i}(t) \approx \mathbf{m}\left(x_{i}, t\right), \mathbf{q}^{i}(t) \approx \mathbf{q}\left(x_{i}, t\right)$. Also define forward and backward difference operators

$$
\delta_{x}^{+} z^{i}=\frac{z^{i+1}-z^{i}}{\xi}, \quad \delta_{x}^{-} z^{i}=\frac{z^{i}-z^{i-1}}{\xi} .
$$

We isolate the spatial derivative terms in (26)-(28) and discretize using symplectic Euler differencing [9] to obtain

$$
\begin{aligned}
& \delta_{x}^{+} \mathbf{q}^{i}=D \mathbf{m}^{i}+\Omega-2 \Lambda \mathbf{m}^{i}-K_{1}\left(\mathbf{m}^{i}\right) \mathbf{m}_{t}^{i}, \\
& -\delta_{x}^{-} \mathbf{m}^{i}=\mathbf{q}^{i} .
\end{aligned}
$$

This system of differential equations satisfies a semi-discrete multisymplectic conservation law extending the result of [25], in which constant $K$ and $L$ were considered. To see this, define $\mathbf{z}^{i}=$ $\left(m_{1}^{i}, m_{2}^{i}, m_{3}^{i}, q_{1}^{i}, q_{2}^{i}, q_{3}^{i}, \Lambda^{i}\right)^{\mathrm{T}}$, and let $s \in \mathcal{S}^{1}$ parameterize a closed curve in phase space.

For $\kappa$ from (25) one finds the identity

$$
\partial_{t} \kappa\left(\mathbf{z}^{i}, \mathbf{z}_{s}^{i}\right)=\partial_{s} \kappa\left(\mathbf{z}^{i}, \mathbf{z}_{t}^{i}\right)-\mathbf{z}_{s}^{i} \cdot K\left(\mathbf{z}^{i}\right) \mathbf{z}_{t}^{i} .
$$


Define a discrete two-form $\bar{\lambda}$ associated with the spatial operator $L$ by

$$
\bar{\lambda}\left(\mathbf{z}^{i-1}, \mathbf{z}^{i}\right)=\mathbf{m}^{i-1} \cdot \mathbf{q}^{i} .
$$

It is easily checked that

$$
\delta_{x}^{+} \bar{\lambda}\left(\mathbf{z}^{i-1}, \mathbf{z}_{s}^{i}\right)=\partial_{s} \bar{\lambda}\left(\mathbf{z}^{i}, \delta_{x}^{+} \mathbf{z}^{i}\right)-\mathbf{z}_{s}^{i} \cdot L \delta_{x}^{ \pm} \mathbf{z}^{i}, \quad \text { where } \delta_{x}^{ \pm} \mathbf{z}^{i}=\left(\begin{array}{c}
\delta_{x}^{-} \mathbf{m}^{i} \\
\delta_{x}^{+} \mathbf{q}^{i}
\end{array}\right) .
$$

Summing (32) and (33) and integrating around $\mathcal{S}^{1}$ gives

$$
\begin{aligned}
& \oint \partial_{t} \kappa\left(\mathbf{z}^{i}, \mathbf{z}_{s}^{i}\right)+\delta_{x}^{+} \bar{\lambda}\left(\mathbf{z}^{i-1}, \mathbf{z}_{s}^{i}\right) \mathrm{d} s \\
& \quad=\oint\left[\kappa\left(\mathbf{z}^{i}, \mathbf{z}_{t}^{i}\right)+\bar{\lambda}\left(\mathbf{z}^{i}, \delta_{x}^{+} \mathbf{z}^{i}\right)\right]_{s}-\left[\mathbf{z}_{s}^{i} \cdot K\left(\mathbf{z}^{i}\right) \mathbf{z}_{t}^{i}+\mathbf{z}_{s}^{i} \cdot L \delta_{x}^{ \pm} \mathbf{z}^{i}\right] \mathrm{d} s=-\oint \frac{\partial S}{\partial s} \mathrm{~d} s=0,
\end{aligned}
$$

which via Stokes theorem yields a semi-discrete multisymplectic conservation law [2].

This spatial discretization also retains a semi-discrete analog of the local energy conservation law (9), namely:

$$
e_{t}^{i}+\delta_{x}^{+} f^{i}=0, \quad e^{i}=\frac{1}{2}\left(-\left(\mathbf{u}^{i}\right)^{2}+\mathbf{m}^{i} \cdot D \mathbf{m}^{i}+2 \Omega \cdot \mathbf{m}^{i}\right), \quad f_{i}=\mathbf{m}_{t}^{i-1} \cdot \mathbf{u}^{i} .
$$

For a given temporal discretization, the error in local energy conservation can be estimated by the residue, defined as

$$
r^{i, n}=\delta_{t}^{+} e^{i, n}+\delta_{x}^{+} \bar{f}^{i, n}, \quad \bar{f}^{i, n}=\delta_{t}^{+} \mathbf{m}^{i-1, n} \cdot \mathbf{u}^{i, n}
$$

Simply substituting the relation (31) into (30) for $\mathbf{q}^{i}$, pre-multiplying by $\hat{\mathbf{m}}^{i}$ and inserting the time derivative of the constraint $\left|m_{i}(t)\right|^{2}=\left|m_{i}(0)\right|^{2}$ as in (29) gives the semi-discretized equation

$$
\mathbf{m}_{t}^{i}=\mathbf{m}^{i} \times\left[\frac{1}{\xi^{2}}\left(\mathbf{m}^{i+1}-2 \mathbf{m}^{i}+\mathbf{m}^{i-1}\right)+D \mathbf{m}^{i}+\Omega\right],
$$

which is globally defined. This system (with $\xi=1$ ) and its higher dimensional generalizations are referred to as the Lattice Landau-Lifshitz equation [5]. It comprises a Hamiltonian ODE with Hamiltonian

$$
H=\frac{1}{2} \sum_{i} \frac{1}{\xi^{2}}\left|\mathbf{m}^{i+1}-\mathbf{m}^{i}\right|^{2}+\mathbf{m}^{i} \cdot D \mathbf{m}^{i}+2 \Omega \cdot \mathbf{m}^{i},
$$

and a Poisson structure (5) with block-diagonal form

$$
B(\mathbf{m})=\left[\begin{array}{lll}
\ddots & & \\
& \hat{\mathbf{m}}^{i} & \\
& & \ddots
\end{array}\right]
$$

Symplectic and time-reversible integrators for (35) were considered in [7]. A symplectic integrator for the isotropic case $D=I_{3}$ was derived by splitting the sum in (36) according to odd and even $i$, such that the dynamics generated by $H_{\mathrm{odd}}$ and $H_{\text {even }}$ are exactly solvable. Since the exact flow map is symplectic for any Hamiltonian and the composition of symplectic maps is symplectic, the overall method is symplectic. Such splitting methods can be made symmetric, and higher order methods can be contrived [18]. A more efficient method was also derived, based on even-odd splitting of the domain. The resulting scheme is 
not symplectic, but time-reversible, and conserves the energy (36) exactly in the isotropic case. Also considered was the implicit midpoint rule (IM), which for this problem is also not symplectic, but is time-reversible and exactly energy conserving. Due to its implicitness, the IM scheme is suitable for use in very fine discretizations, where the explicit methods suffer from a stability restriction on the stepsize.

Another, possibly better, explicit splitting method is based on a three-term splitting of the Hamiltonian into $m_{1}, m_{2}$ and $m_{3}$ contributions:

$$
H=H_{1}+H_{2}+H_{3}, \quad H_{j}=\frac{1}{2} \sum_{i} \frac{1}{\xi^{2}}\left(m_{j}^{i+1}-m_{j}^{i}\right)^{2}+d_{j}\left(m_{j}^{i}\right)^{2}+2 \Omega_{j} m_{j}^{i} .
$$

The dynamics generated by $H_{1}$, for example, are

$$
\partial_{t}\left(\begin{array}{l}
m_{1}^{i} \\
m_{2}^{i} \\
m_{3}^{i}
\end{array}\right)=\left[\begin{array}{ccc}
0 & -m_{3}^{i} & m_{2}^{i} \\
m_{3}^{i} & 0 & -m_{1}^{i} \\
-m_{2}^{i} & m_{1}^{i} & 0
\end{array}\right]\left(\begin{array}{c}
\frac{\partial H_{1}}{\partial m_{1}^{i}} \\
0 \\
0
\end{array}\right)=\left(\begin{array}{c}
0 \\
\frac{\partial H_{1}}{\partial m_{1}^{i}} m_{3}^{i} \\
-\frac{\partial H_{1}}{\partial m_{1}^{i}} m_{2}^{i}
\end{array}\right),
$$

which is easily solved to give a rotation about the $m_{1}$ axis. The dynamics due to $H_{2}$ and $H_{3}$ are analogous. Let $\Phi_{\tau, j}$ represent the solution operator for the dynamics due to $H_{j}$ over an interval $\tau$. The symmetric composition method

$$
\mathbf{m}^{n+1}=\Phi_{\tau / 2,1} \circ \Phi_{\tau / 2,2} \circ \Phi_{\tau, 3} \circ \Phi_{\tau / 2,2} \circ \Phi_{\tau / 2,1} \mathbf{m}^{n},
$$

is second order and symplectic [18]. This method has been used by a number of authors to integrate the Euler rigid body equations (see, e.g., [17]). Its main advantages over the methods of [7] are that it is both fast and symplectic (though not exactly energy conserving), and it allows a uniform treatment of anisotropy.

To understand how this splitting fits into the multisymplectic framework, define a decomposition $L=L_{1}+L_{2}+L_{3}$ of the spatial symplectic operator, with the nonzero components of $L_{j}$ given by $\left(L_{j}\right)_{j, j+3}=1=-\left(L_{j}\right)_{j+3, j}$, and associated symplectic 2-form $\bar{\lambda}_{j}$. Similarly, let $S_{j}(\mathbf{z})=\frac{1}{2}\left(q_{j}^{2}+d_{j} m_{j}^{2}+\right.$ $\left.2 \Omega_{j} m_{j}\right)-\Lambda\left(|\mathbf{m}|^{2}-m_{\ell}^{2}\right)$. Then the split flows $K\left(\mathbf{z}^{i}\right) \mathbf{z}_{t}^{i}+L_{j} \delta_{x}^{ \pm} \mathbf{z}^{i}=S_{j}\left(\mathbf{z}^{i}\right)$ are solved consecutively and exactly in time, yielding a sequence of semi-discrete multisymplectic conservation laws

$$
\partial_{t} \kappa\left(\mathrm{d} \mathbf{z}_{j}^{i}, \mathrm{~d} \mathbf{z}_{j}^{i}\right)+\delta_{x}^{+} \bar{\lambda}_{j}\left(\mathrm{~d} \mathbf{z}_{j}^{i-1}, \mathrm{~d} \mathbf{z}_{j}^{i}\right)=0, \quad j=1,2,3,
$$

analogous to (16), where the differential $\mathrm{d} \mathbf{z}_{j}$ solves the variational equation associated with the $j$ th flow. Summing these relations across the grid with periodic boundary conditions shows that each split timestep is globally symplectic, implying that the composite time integrator is globally symplectic, however it is not clear to what extent the composition may be interpreted as a local conservation law of symplecticity in the sense of [3]. There exist splittings that clearly preserve local conservation, but these are restricted to Hamiltonian splittings for which the identity (27) remains intact, which for the LLE essentially means solving the exact dynamics. Besides splitting, other options for obtaining symplectic integrators for the structure (37) include seeking a global transformation to canonical form or Lie group integrators [9]. Recent papers on Lie group integrators for Landau-Lifshitz equations are [10,13].

Instead, in the next section we will drop the requirement of multisymplecticity and focus on the energy conservation law. 


\section{Box scheme discretization}

Bridges and Reich [3] proposed the multisymplectic box scheme and showed that it preserves discrete energy and momentum conservation laws analogous to (9), (10) for multisymplectic PDEs with quadratic Hamiltonians. For constant symplectic operators $K$ and $L$, such PDEs are linear. For the LLE the box scheme is no longer symplectic in time, i.e., it is not a Poisson map for the symplectic operator $K(\mathbf{z})$ of (24). However, since the Hamiltonian (23) is quadratic and $L$ is constant, a discrete energy conservation law still holds. The discrete momentum law is also lost due to the nonlinearity of $K(\mathbf{z})$.

Let $\mathbf{z}^{i, n} \approx \mathbf{z}\left(x_{i}, t_{n}\right)$ and define, for an arbitrary function $f$, the average and difference operators

$$
\begin{array}{rlrl}
\mu_{x} \mathbf{z}^{i, n} & =\frac{1}{2}\left(\mathbf{z}^{i+1, n}+\mathbf{z}^{i, n}\right), & \delta_{x} \mathbf{z}^{i, n}=\frac{1}{\xi}\left(\mathbf{z}^{i+1, n}-\mathbf{z}^{i, n}\right), \\
\mu_{t} \mathbf{z}^{i, n}=\frac{1}{2}\left(\mathbf{z}^{i, n+1}+\mathbf{z}^{i, n}\right), & \delta_{t} \mathbf{z}^{i, n}=\frac{1}{\tau}\left(\mathbf{z}^{i, n+1}-\mathbf{z}^{i, n}\right),
\end{array}
$$

all of which mutually commute. Using these definitions, a discrete chain rule holds for bilinear forms $\beta(\mathbf{v}, \mathbf{w})$ :

$$
\begin{aligned}
& \beta\left(\delta_{x} \mathbf{v}^{i}, \mu_{x} \mathbf{w}^{i}\right)+\beta\left(\mu_{x} \mathbf{v}^{i}, \delta_{x} \mathbf{w}^{i}\right) \\
& =\frac{1}{2 \xi}\left[\beta\left(\mathbf{v}^{i+1}, \mathbf{w}^{i+1}\right)+\beta\left(\mathbf{v}^{i}, \mathbf{w}^{i+1}\right)-\beta\left(\mathbf{v}^{i+1}, \mathbf{w}^{i}\right)-\beta\left(\mathbf{v}^{i}, \mathbf{w}^{i}\right)\right. \\
& \left.\quad+\beta\left(\mathbf{v}^{i+1}, \mathbf{w}^{i+1}\right)-\beta\left(\mathbf{v}^{i}, \mathbf{w}^{i+1}\right)+\beta\left(\mathbf{v}^{i+1}, \mathbf{w}^{i}\right)-\beta\left(\mathbf{v}^{i}, \mathbf{w}^{i}\right)\right] \\
& =\frac{1}{\xi}\left[\beta\left(\mathbf{v}^{i+1}, \mathbf{w}^{i+1}\right)-\beta\left(\mathbf{v}^{i}, \mathbf{w}^{i}\right)\right]=\delta_{x} \beta\left(\mathbf{v}^{i}, \mathbf{w}^{i}\right) .
\end{aligned}
$$

The same relations hold for $\mu_{t}$ and $\delta_{t}$.

Consider the multisymplectic form with nonconstant temporal symplectic operator and quadratic function $S(\mathbf{z})=\frac{1}{2} \mathbf{z} \cdot A \mathbf{z}$ :

$$
K(\mathbf{z}) \mathbf{z}_{t}+L \mathbf{z}_{x}=A \mathbf{z} .
$$

The box scheme discretization for this system is

$$
K\left(\mu_{x} \mu_{t} \mathbf{z}^{i, n}\right) \delta_{t} \mu_{x} \mathbf{z}^{i, n}+L \delta_{x} \mu_{t} \mathbf{z}^{i, n}=A \mu_{x} \mu_{t} \mathbf{z}^{i, n} .
$$

Computing the inner product of this expression with $\delta_{t} \mu_{x} \mathbf{z}^{i, n}$, and using the skew-symmetry of $K(\mathbf{z})$, we obtain

$$
\delta_{t} \mu_{x} \mathbf{z}^{i, n} \cdot L \delta_{x} \mu_{t} \mathbf{z}^{i, n}=\delta_{t} \mu_{x} \mathbf{z}^{i, n} \cdot A \mu_{x} \mu_{t} \mathbf{z}^{i, n} .
$$

The left side of this equation is, using (39) and skew-symmetry of $L$,

$$
\begin{aligned}
& \delta_{t} \mu_{x} \mathbf{z}^{i, n} \cdot L \mu_{t} \delta_{x} \mathbf{z}^{i, n}=\frac{1}{2} \delta_{t}\left(\mu_{x} \mathbf{z}^{i, n}\right) \cdot L \mu_{t}\left(\delta_{x} \mathbf{z}^{i, n}\right)+\frac{1}{2} \mu_{x}\left(\delta_{t} \mathbf{z}^{i, n}\right) \cdot L \delta_{x}\left(\mu_{t} \mathbf{z}^{i, n}\right) \\
& =\frac{1}{2} \delta_{x}\left(\delta_{t} \mathbf{z}^{i, n} \cdot L \mu_{t} \mathbf{z}^{i, n}\right)-\frac{1}{2} \delta_{x} \delta_{t} \mathbf{z}^{i, n} \cdot L \mu_{x} \mu_{t} \mathbf{z}^{i, n}+\frac{1}{2} \delta_{t}\left(\mu_{x} \mathbf{z}^{i, n} \cdot L \delta_{x} \mathbf{z}^{i, n}\right)-\frac{1}{2} \mu_{x} \mu_{t} \mathbf{z}^{i, n} \cdot L \delta_{x} \delta_{t} \mathbf{z}^{i, n} \\
& =\frac{1}{2} \delta_{t}\left(\mu_{x} \mathbf{z}^{i, n} \cdot L \delta_{x} \mathbf{z}^{i, n}\right)+\frac{1}{2} \delta_{x}\left(\delta_{t} \mathbf{z}^{i, n} \cdot L \mu_{t} \mathbf{z}^{i, n}\right),
\end{aligned}
$$


and the right side is, using (39) and symmetry of $A$,

$$
\delta_{t} \mu_{x} \mathbf{z}^{i, n} \cdot A \mu_{x} \mu_{t} \mathbf{z}^{i, n}=\frac{1}{2} \delta_{t}\left(\mu_{x} \mathbf{z}^{i, n} \cdot A \mu_{x} \mathbf{z}^{i, n}\right) .
$$

Combining the last two relations gives the desired discrete energy conservation law

$$
\delta_{t}\left(\mu_{x} \mathbf{z}^{i, n} \cdot L \delta_{x} \mathbf{z}^{i, n}-\mu_{x} \mathbf{z}^{i, n} \cdot A \mu_{x} \mathbf{z}^{i, n}\right)+\delta_{x}\left(\delta_{t} \mathbf{z}^{i, n} \cdot L \mu_{t} \mathbf{z}^{i, n}\right)=0 .
$$

For the specific case (26)-(28) discretization with the box scheme gives

$$
\begin{aligned}
& K_{1}\left(\mu_{t} \mu_{x} \mathbf{m}^{i, n}\right) \delta_{t} \mu_{x} \mathbf{m}^{i, n}+\delta_{x} \mu_{t} \mathbf{q}^{i, n}=D \mu_{t} \mu_{x} \mathbf{m}^{i, n}+\Omega-2 \Lambda \mu_{t} \mu_{x} \mathbf{m}^{i, n}, \\
& -\delta_{x} \mu_{t} \mathbf{m}^{i, n}=\mu_{t} \mu_{x} \mathbf{q}^{i, n}, \\
& 0=\left|\mu_{t} \mu_{x} \mathbf{m}^{i, n}\right|^{2}-m_{\ell}\left(x_{i}+\xi / 2,0\right)^{2} .
\end{aligned}
$$

For a numerical implementation of (41)-(43), we premultiply (42) by $\delta_{x} \mu_{x}^{-1}$ and substitute into (41) to eliminate $\mathbf{q}^{i, n}$. We then premultiply both sides by $\mu_{t} \mu_{x} \hat{\mathbf{m}}^{i, n}$ and substitute the discrete derivative of (43) as in the continuous case. Because (43) enforces the spin length constraint at $x_{i}+\xi / 2$, we prefer to work with the spatially averaged spin $\overline{\mathbf{m}}^{i, n}=\mu_{x} \mathbf{m}^{i, n}$, for which the method becomes

$$
\delta_{t} \overline{\mathbf{m}}^{i, n}=\mu_{t} \overline{\mathbf{m}}^{i, n} \times\left[\left(\delta_{x} \mu_{x}^{-1}\right)^{2} \mu_{t} \overline{\mathbf{m}}^{i, n}+D \mu_{t} \overline{\mathbf{m}}^{i, n}+\Omega\right],
$$

which is an implicit midpoint update. The operator $\mu_{x}^{-1}$ exists for periodic boundary conditions and number of gridpoints $N$ odd. For $N$ even, $\mu_{x}$ can be inverted up to the alternating grid sequence using the pseudoinverse.

\section{Numerical verification}

In this section, we provide a preliminary evaluation of the new methods on the basis of numerical experiments.

All numerical experiments utilize the soliton solution to the LLE published by Tjon and Wright [26]. The soliton is defined, for the anisotropic $\operatorname{LLE}(D=I)$, by

$$
m_{1}(\eta)=\sin \theta(\eta) \cos \phi(\eta), \quad m_{2}(\eta)=\sin \theta(\eta) \sin \phi(\eta), \quad m_{3}=\cos \theta(\eta),
$$

where $\eta=x-x_{0}-V t$ and

$$
\begin{aligned}
& \cos \theta(\eta)=1-2 b^{2} \operatorname{sech}^{2}(b \sqrt{\omega} \eta), \\
& \phi(\eta)=\frac{1}{2} V\left(x-x_{0}\right) \pm \tan ^{-1}\left[\left(\frac{b^{2}}{1-b^{2}}\right)^{1 / 2} \tanh (b \sqrt{\omega} \eta)\right],
\end{aligned}
$$

and the parameters $V, \omega$, and $b$ satisfy $V^{2} /(4 \omega)=1-b^{2} . V$ is the translation speed of the soliton, $b$ determines its size, and the sign \pm in (45) should agree with that of $V$. With the external magnetic field given by $\Omega=\left(0,0, \Omega_{3}\right)^{\mathrm{T}}$, the parameter $\omega$ in (44)-(45) satisfies $\omega=\Omega_{3}+\omega_{0}$, with $\omega_{0}$ determining the relative phase of $m_{1}$ and $m_{2}$. These equations describe a right-running wave for positive $V$ and a left running wave for negative $V$. The function $1-m_{3}(\eta)$ is a "pulse" centered at $\eta=0$. The soliton solution is defined on the whole real line, but we have truncated it and use periodic boundary conditions on a domain of length $48 \pi$. 


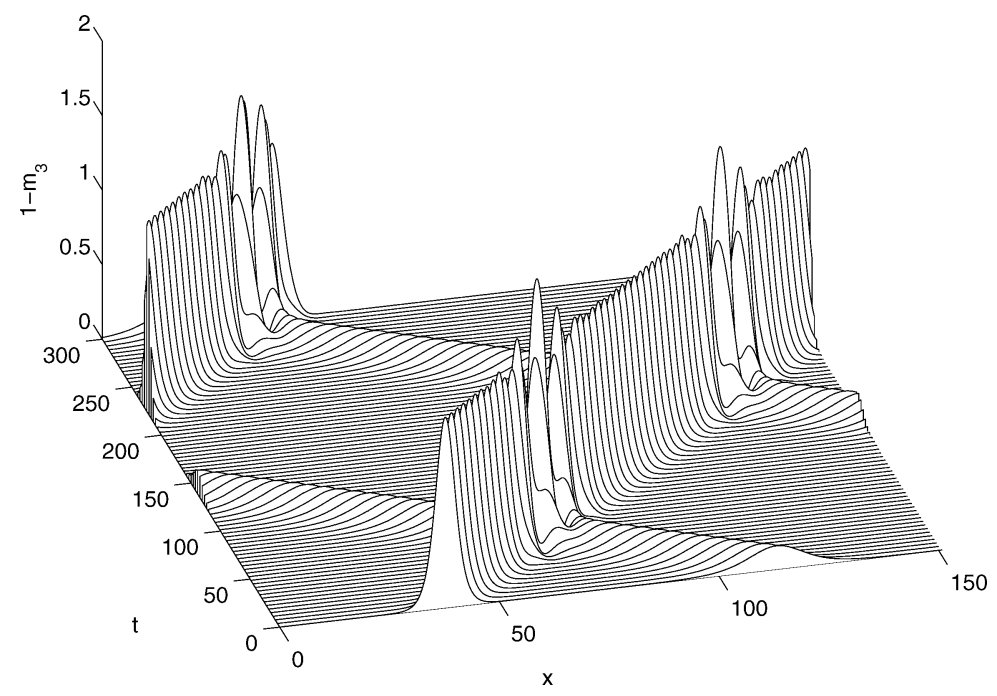

Fig. 1. Collision of two solitons computed with the splitting method $(N=600, \tau=0.01)$.

Splitting, $\mathrm{N}=100$

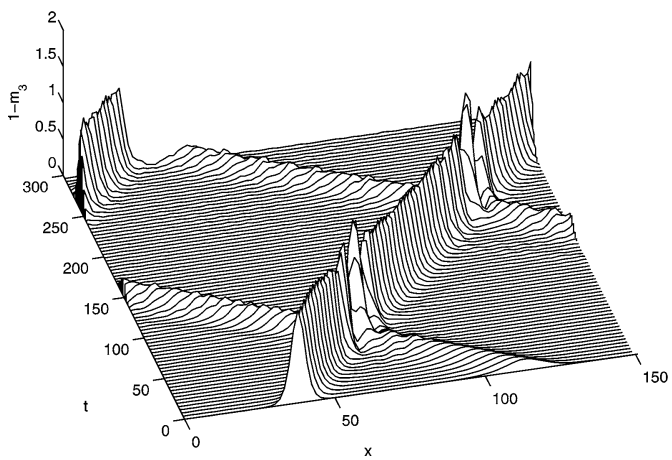

Box, $N=100$

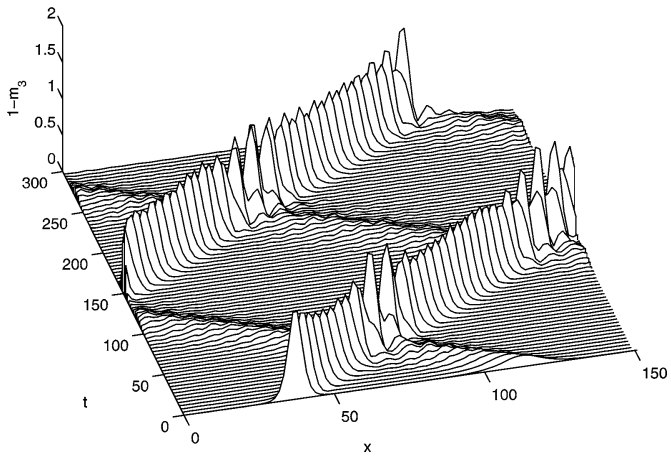

Fig. 2. Collision of two poorly resolved solitons computed with the splitting method (left) and box scheme (right), $N=100$, $\tau=0.3$.

To simulate a two-soliton collision we chose parameters

$$
V_{1}=0.5, \quad b_{1}=0.8, \quad V_{2}=-0.8, \quad \Omega_{3}=\omega_{2}=\omega_{1},
$$

for which $b_{2}=0.28$. The two solitons were initially located at $x_{1}=12 \pi$ and $x_{2}=36 \pi$.

The LLE was discretized on a grid with $N$ grid points and periodic boundary conditions using the splitting method (38) and the box scheme (41)-(43). The methods were implemented in Matlab, and for the box scheme Newton iterations were done at time level $n+1$ using the Jacobian from time level $n$, until convergence of the residue to $10^{-13}$ in the maximum norm.

Fig. 1 illustrates the dynamics of the pulse-like component $1-m_{3}$ through approximately one period of motion $([0,300])$ of the slow soliton, computed using the splitting method at grid resolution $N=600$. To more clearly distinguish the features of the two methods, a poorly resolved discretization on $N=100$ grid points was simulated over the same time interval. Fig. 2 illustrates the comparison. The solution 

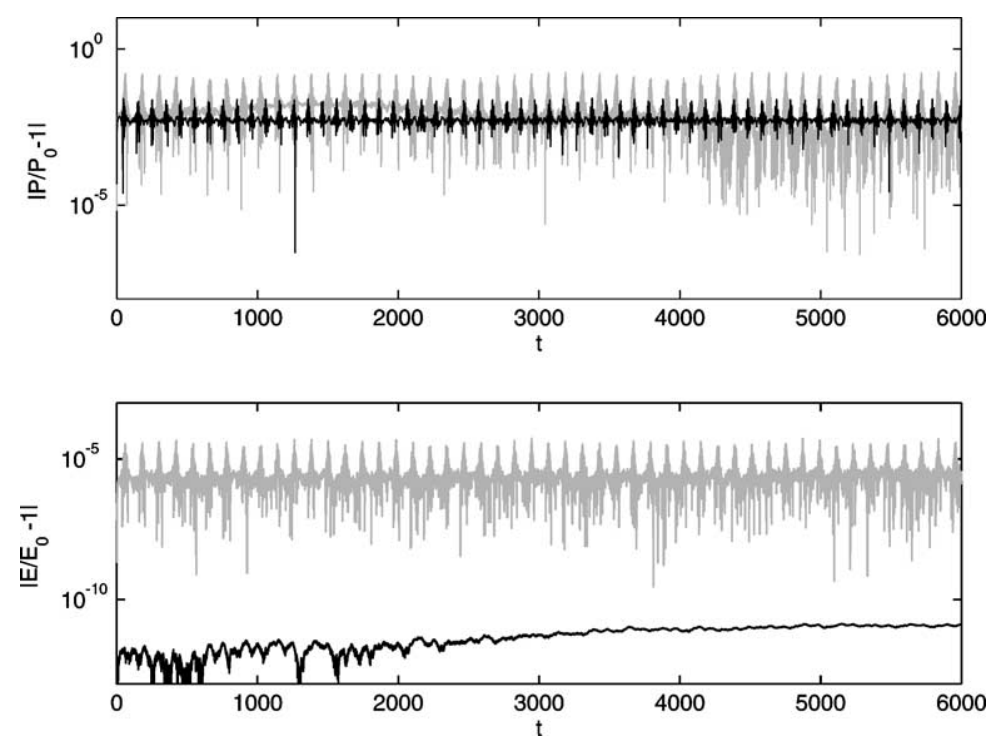

Fig. 3. Relative change in total momentum (top) and total energy (bottom) for a long simulation of 50 soliton collisions, splitting method (gray) and box scheme (black), $N=150, \tau=0.2, T=6000$.

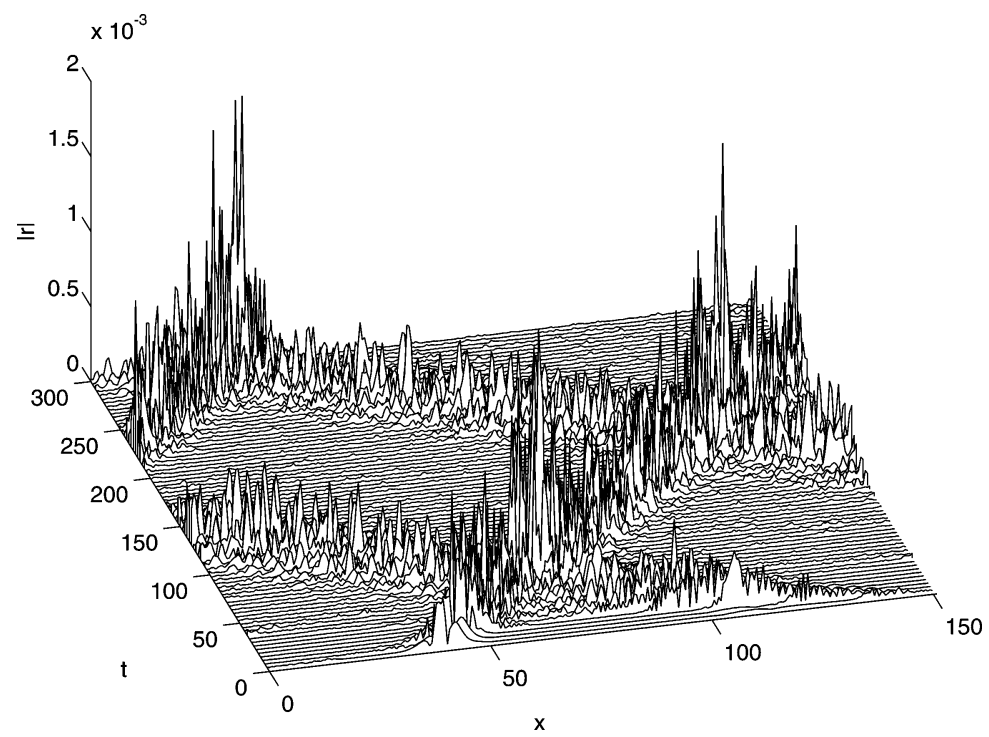

Fig. 4. Residue in local energy conservation law (34) for the splitting method. $N=300, \tau=0.05$.

obtained with the splitting method exhibits a small lag in group velocity compared to the more accurate solution in Fig. 1. The box scheme has a more severe, accelerated group velocity: at the current grid resolution, the slow soliton evolves through approximately 1.5 periods. Comparing the quality of the two solutions, the splitting method is smoother but tends to deteriorate as the integration progresses, and appears to support some small reflected waves emanating from the collision. With the box scheme, 
especially the small soliton is very poorly resolved for this grid size, but appears to stabilize before the first collision. No reflections are observed.

We also carried out a long simulation through more than 50 collisions to compare the global conservation properties of the two methods. Using a mesh with $N=150$, both methods were integrated with $\tau=0.2$ on an interval [0,6000]. Fig. 3 shows the relative changes in total momentum and total energy. Both quantities were well-conserved by the splitting method. For the box scheme the total energy is exactly conserved up to truncation error of the Newton iteration. For the given tolerance $\left(10^{-13}\right)$ there is a small drift of magnitude $10^{-11}$. Total momentum is not exactly conserved, but the peaks in momentum error with the box scheme are smaller by a factor 10 than those obtained with the splitting method.

The conservation of total energy for the box scheme is a consequence of the exact preservation of the discrete local conservation law (40) under periodic boundary conditions. We also estimate the error in local energy conservation incurred by the splitting method by plotting the absolute value of the residue (34) in Fig. 4 for $N=300$. From the figure it is evident that the residue is largest near the solitons, and that the peaks observed in Fig. 3 are accompanied by larger local residues near collisions, but that there are small peaks in the quiescent regions as well. The change in total energy, obtained by summing the $r^{i, n}$ over $i$, is an order of magnitude smaller than the local quantity due to cancellation of positive and negative contributions. As $\tau$ tends to zero, the amplitudes of the peaks in Fig. 4 converge to zero.

\section{Conclusions and extensions}

In this paper we have generalized the idea of multisymplectic structure to the nonlinear case of the Landau-Lifshitz equation. Motivated by this structure we have proposed a new box scheme discretization which, though not multisymplectic, does retain a discrete energy conservation law. We have also shown that the standard discretization leads to a semi-discrete multisymplectic PDE, which in turn can be discretized in space using a globally symplectic splitting method.

The methods presented both give good behavior for soliton collisions. The splitting method is globally symplectic and very fast. The box scheme satisfies the discrete analog of the implicit energy conservation law, implying exact global energy conservation, and appears to conserve total momentum better as well. The implications of local energy conservation need to be investigated further.

In micromagnetics applications, the LLE is often coupled with an external field satisfying Maxwell's equations [6]. These equations also have a simple multisymplectic structure, suggesting a unified approach. Maxwell's equations are, for $\mathbf{E}$ the electric field and $\mathbf{B}$ the magnetic induction,

$$
\mathbf{B}_{t}=\nabla \times \mathbf{E}, \quad-\mathbf{E}_{t}=\nabla \times \mathbf{B}, \quad \nabla \cdot \mathbf{B}=\nabla \cdot \mathbf{E}=0 .
$$

Writing $\mathbf{z}^{\mathrm{T}}=\left(\mathbf{E}^{\mathrm{T}}, \mathbf{B}^{\mathrm{T}}\right)$, Maxwell's equations assume the three-dimensional multisymplectic structure $K \mathbf{z}_{t}+L^{1} \mathbf{z}_{x}+L^{2} \mathbf{z}_{y}+L^{3} \mathbf{z}_{z}=0$ with

$$
\begin{array}{rlr}
K= & {\left[\begin{array}{cc}
0 & I \\
-I & 0
\end{array}\right],} & L^{j}=\left[\begin{array}{cc}
\sigma_{j} & 0 \\
0 & \sigma_{j}
\end{array}\right], \\
\sigma_{1}=\left[\begin{array}{ccc}
0 & 0 & 0 \\
0 & 0 & -1 \\
0 & 1 & 0
\end{array}\right], & \sigma_{2}=\left[\begin{array}{ccc}
0 & 0 & 1 \\
0 & 0 & 0 \\
-1 & 0 & 0
\end{array}\right], & \sigma_{3}=\left[\begin{array}{ccc}
0 & -1 & 0 \\
1 & 0 & 0 \\
0 & 0 & 0
\end{array}\right] .
\end{array}
$$


Both the box scheme and the symplectic Euler discretization could be applied here, and the box scheme would satisfy discrete conservation laws of symplecticity and energy as well as momenta in 3 directions.

\section{Acknowledgements}

Thanks are due to G. Bertotti of the Istituto Elettrotecnico Nazionale of Turin, Italy for help with the variational formulation of the Landau-Lifshitz equation and to S. Reich of the Imperial College of London for invaluable discussions and suggestions. Suggestions of the referees also helped to clarify several points.

\section{References}

[1] T.J. Bridges, Multi-symplectic structures and wave propagation, Math. Proc. Cambridge Philos. Soc. 121 (1997) 147-190.

[2] T.J. Bridges, A geometric formulation of the conservation of wave action and its implications for signature and the classification of instabilities, Proc. Roy. Soc. London A 453 (1997) 1365-1395.

[3] T.J. Bridges, S. Reich, Multi-symplectic integrators: numerical schemes for Hamiltonian PDEs that conserve symplecticity, Phys. Lett. A 284 (2001) 184-193.

[4] W. E, X.-P. Wang, Numerical methods for the Landa-Lifshitz equation, SIAM J. Numer. Anal. 38 (2000) $1647-1665$.

[5] L.D. Faddeev, L.A. Takhtajan, Hamiltonian Methods in the Theory of Solitons, Springer, Berlin, 1980.

[6] J. Fidler, T. Schrefl, Micromagnetic modelling-the current state of the art, J. Phys. D: Appl. Phys. 33 (2000) R135-R156.

[7] J. Frank, W. Huang, B. Leimkuhler, Geometric integrators for classical spin systems, J. Comput. Phys. 133 (1997) 160172.

[8] E. van Groesen, E.M. de Jager, Mathematical Structures in Continuous Dynamical Systems, in: Stud. Math. Phys., vol. 6, Elsevier, Amsterdam, 1994.

[9] E. Hairer, C. Lubich, G. Wanner, Geometric Numerical Integration: Structure Preserving Algorithms for Ordinary Differential Equations, Springer, Berlin, 2002.

[10] P.S. Krishnaprasad, X. Tan, Cayley transforms in micromagnetics, Physica B 306 (2001) 195-199.

[11] C. Lanczos, The Variational Principles of Mechanics, fourth ed., Dover, New York, 1986.

[12] A. Lew, J.E. Marsden, M. Ortiz, M. West, Asynchronous variational integrators, Arch. Rational Mech. Anal. 167 (2003) 85-146.

[13] D. Lewis, N. Nigam, Geometric integration on spheres and some interesting applications, J. Comput. Appl. Math. 151 (2003) 141-170.

[14] J.E. Marsden, G.W. Patrick, S. Shkoller, Multisymplectic geometry, variational integrators, and nonlinear PDEs, Comm. Math. Phys. 199 (1999) 351-395.

[15] J.E. Marsden, S. Pekarsky, S. Shkoller, M. West, Variational methods, multisymplectic geometry and continuum mechanics, J. Geom. Phys. 38 (2001) 253-284.

[16] J.E. Marsden, T.S. Ratiu, Introduction to Mechanics and Symmetry, Springer, Berlin, 1994.

[17] R.I. McLachlan, Explicit Lie-Poisson integration and the Euler equations, Phys. Rev. Lett. 71 (1993) $3043-3046$.

[18] R.I. McLachlan, G.R.W. Quispel, Splitting methods, Acta Numer. (2002) 341-434.

[19] F.G. Mertens, A.R. Bishop, Dynamics of vortices in two-dimensional magnets, in: P.L. Christiansen, M.P. Sorensen (Eds.), Nonlinear Science at the Dawn of the 21st Century, Springer, Berlin, 1999.

[20] P.B. Monk, O. Vacus, Error estimates for a numerical scheme for ferromagnetic problems, SIAM J. Numer. Anal. 36 (1999) 696-718.

[21] P.B. Monk, O. Vacus, Accurate discretization of a non-linear micromagnetic problem, Comput. Methods Appl. Mech. Engrg. 190 (2001) 5243-5269.

[22] P.J. Olver, Applications of Lie Groups to Differential Equations, second ed., Springer, Berlin, 1993.

[23] N. Papanicolaou, P.N. Spathis, Semitopological solitions in planar ferromagnets, Nonlinearity 12 (1999) $285-302$.

[24] G.W. Patrick, The Landau-Lifshitz equation by semi-direct product reduction, Lett. Math. Phys. 50 (1999) $177-188$.

[25] S. Reich, Multi-symplectic Runge-Kutta collocation methods for Hamiltonian wave equations, J. Comput. Phys. 157 (2000) 473-499.

[26] J. Tjon, J. Wright, Solitons in the continuous Heisenberg spin chain, Phys. Rev. B 15 (1977) 3470-3476. 